\title{
A Broadband Microwave Radiometer Technique at X-band for Rain and Drop Size Distribution Estimation
}

\author{
R. Meneghini, Senior Member, IEEE
}

\begin{abstract}
Radiometric brightness temperatures below about 12 $\mathrm{GHz}$ provide accurate estimates of path attenuation through precipitation and cloud water. Multiple brightness temperature measurements at $\mathrm{X}$-band frequencies can be used to estimate rainfall rate and parameters of the drop size distribution once correction for cloud water attenuation is made. Employing a stratiform storm model, calculations of the brightness temperatures at $9.5,10$ and 12 $\mathrm{GHz}$ are used to simulate estimates of path-averaged median mass diameter, number concentration and rainfall rate. The results indicate that reasonably accurate estimates of rainfall rate and information on the drop size distribution can be derived over ocean under low to moderate wind speed conditions.
\end{abstract}

Index Terms-airborne radiometer, drop size distribution, microwave radiometry, rain rate estimation, spaceborne radiometer

\section{INTRODUCTION}

For frequencies up to about $12 \mathrm{GHz}$, the radiometric brightness temperature from rain over ocean is closely related to the path integrated attenuation (PIA) for low to moderate surface wind speeds. Multi-frequency measurements of brightness temperature at $\mathrm{X}$-band $(8.2-12.4 \mathrm{GHz})$ over a bandwidth of $2-3 \mathrm{GHz}$, in principle, provide sufficient information to estimate parameters of the drop size distribution and rainfall rate. The purpose of this paper is to derive equations for and investigate the feasibility of this type of parameter estimation.

The instrument concept is similar to that used in the steppedfrequency microwave radiometer (SFMR). In the SFMR, multiple bands in the frequency range from 4.6 to $7.2 \mathrm{GHz}$ are used to estimate rainfall rate and near-surface wind speed over the ocean $[1,2]$. Here, the emphasis is different in the sense that the objective is to estimate parameters of the pathaveraged drop size distribution (DSD) and rain rate using Xband frequencies. The approach considered here is also related to that used for estimation of DSD parameters from multi-frequency transmission measurements along a microwave link [3-5]. In essence, two path-attenuation measurements yield two parameters of the exponential form of the DSD or, equivalently, two parameters of the gamma DSD

Manuscript received April 2, 2004. R. Meneghini is with NASA.Goddard Space Flight Center, Code 975, Greenbelt, MD 20771. (Ph: 301-614-5652; fax: 301-614-5558, email: Robert.Meneghini-1@nasa.gov). with the shape parameter, $\mu$, fixed or expressed as a function of one of the variable parameters [6]. There are, however, several complicating factors in the application of this technique to microwave radiometry. The first is that conversion of brightness temperature, $T_{B}$, to path-integrated attenuation, $\mathrm{A}$, is not one-to-one: changes in the characteristics of the mixed-phase particles and scattering contributions from the ice and water introduce uncertainty in the conversion of $T_{B}$ to A that translate into estimation errors. Cloud liquid water presents a somewhat different problem. Cloud droplets are Rayleigh scatterers/absorbers within the frequency band of interest so that the functional dependence of the specific attenuation on frequency is known. This contribution can be eliminated in part by considering the difference of path attenuations with suitable normalizations. Once the rain parameters are estimated from differential quantities, the cloud liquid water, in principle, can be recovered from the equation for total path attenuation.

For operation below or above $\mathrm{X}$-band, the error sources become larger. Below X-band, the differential attenuation is typically small and the relative errors in the estimate render the estimates inaccurate. While the differential attenuation is relatively strong above $12 \mathrm{GHz}$, greater scattering contributions to $T_{B}$ from ice and snow, as well as the rain itself, introduce an increasing amount of variability into the $T_{B}$ - A relationship, making the estimation of DSD parameters impractical.

The use of closely-spaced frequencies has also been considered for airborne and spaceborne weather radars $[7,8]$. For radar, the differential reflectivity factor serves as an estimator of the median mass diameter, $D_{0}$, of rain as well as snow. For the X-band radiometer, a ratio of normalized differential path attenuations, derived from brightness temperatures, provides an estimator for the path-averaged median mass diameter of the rain. In a sense, the radiometerbased algorithm is more straightforward: for the radar application, correction for attenuation must be made before the differential reflectivity can be used whereas for the radiometer algorithm, a function of the differential attenuation serves as a direct estimator of the path-averaged $D_{0}$ in rain. As in most applications of air- or spaceborne radar and radiometer to precipitation, the primary advantage of the radar is its rangeprofiling capability while the attractions of the microwave 
radiometer are higher reliability, lower cost, and, typically, more rapid scanning capabilities.

\section{ALGORITHM CONSIDERATIONS}

The first objective is to express parameters of the DSD and rain rate as functions of the PIA or differential PIA. In the following section, these quantities are related to the brightness temperatures so that a connection is made between the measurements and the quantities to be estimated.

Let $\widetilde{A}(f)$ be the PIA (dB) at frequency $f(\mathrm{~Hz})$ from the storm top (range $\mathrm{r}=0)$ to the surface $\left(\mathrm{r}=\mathrm{r}_{\mathrm{s}}\right)$ and let $k(f, s)$ be the specific attenuation $\left(\mathrm{dB} \mathrm{km}^{-1}\right)$ at $\mathrm{r}=\mathrm{s}$. The quantities $\widetilde{A}(f)$ and $k(f, s)$ are related by:

$$
\tilde{A}(f)=\int_{0}^{r_{s}} k(f, s) d s
$$

The specific attenuation consists of terms corresponding to contributions from rain, snow, mixed-phase precipitation as well as cloud liquid and cloud ice and various atmospheric gases such as water vapor and oxygen. Although the effects of atmospheric gases will be assessed in the error analysis given later, for the purpose of constructing estimators of the DSD parameters, we assume that the contributions from rain and mixed-phase precipitation, $k_{p}$, and that from cloud liquid water, $k_{c}$, dominate so that

$$
k=k_{c}+k_{p}
$$

As the cloud water droplets much smaller than the wavelengths of interest, $k_{c}$ can be related to the cloud water content, $M_{c}(\mathrm{~g}$ $\mathrm{m}^{-3}$ ) by [9]

$$
k_{c}=[(0.4343 \times 6 \pi f) / c] \operatorname{Im}(-K) M_{c}
$$

where $\mathrm{c}$ is the speed of light $\left(\mathrm{cm} \mathrm{s}^{-1}\right)$ and $K$ is the dielectric factor related to the complex index of refraction of water, $m$, by:

$$
K=\frac{m^{2}-1}{m^{2}+2}
$$

The imaginary part of the dielectric factor, $\operatorname{Im}(-K)$, is a function of frequency and temperature, $T$, or height. To remove, in an approximate sense, the influence of cloud water on the DSD and rain rate estimates, consider the following two normalization factors:

$$
\begin{aligned}
& \alpha_{1}(f)=[(0.4343 \times 6 \pi f) / c] \\
& \alpha_{2}(f, \bar{T})=[(0.4343 \times 6 \pi f) / c] \operatorname{Im}(-K(f, \bar{T}))
\end{aligned}
$$

where $\bar{T}$ in (5b) is the estimated mean temperature of the cloud droplets. Normalizing (3) by $\alpha_{n}$ gives:

$$
k_{c} / \alpha_{n}=q_{n} M_{c}
$$

where $\mathrm{n}=1$ or 2 and where

$q_{1}=\operatorname{Im}(-K(f, T))$

$q_{2}=[\operatorname{Im}(-K(f, T) / \operatorname{Im}(-K(f, \bar{T}))]$

Dividing $\tilde{A}(f)$ by $\alpha_{n}$ and letting $\tilde{A}(f) / \alpha_{n}=A_{n}(f)$ then, on using (1)-(3), the normalized path attenuation can be written

$$
A_{n}(f)=\int_{0}^{r_{s}}\left[\alpha_{n}^{-1}(f, \bar{T}) k_{p}(f, s)+q_{n}(f, T, \bar{T}) M_{c}(s)\right] d s
$$

If brightness temperature measurements are available at frequencies $\left.f_{j}, f_{i}\left(f_{j}>f_{i}\right)\right)$ then the difference of the normalized path attenuations can be written:

$$
\delta A_{n}\left(f_{j}, f_{i}\right) \equiv \alpha_{n}^{-1}\left(f_{j}, \bar{T}\right) \tilde{A}\left(f_{j}\right)-\alpha_{n}^{-1}\left(f_{i}, \bar{T}\right) \tilde{A}\left(f_{i}\right)
$$

Assuming for either value of $\mathbf{n}$ that

$$
\int_{0}^{r_{s}}\left[q_{n}\left(f_{j}, T, \bar{T}\right)-q_{n}\left(f_{i}, T, \bar{T}\right)\right] M_{c}(s) d s \approx 0
$$

then an approximation for the normalized differential path attenuation, independent of cloud liquid water, is:

$$
\mathcal{A}_{n}\left(f_{j}, f_{i}\right) \cong \int_{0}^{r_{s}}\left[\alpha_{n}^{-4}\left(f_{j}, \bar{T}\right) k_{p}\left(f_{j}, s\right)-\alpha_{n}^{-1}\left(f_{i}, \bar{T}\right) k_{p}\left(f_{i}, s\right)\right] d s
$$

To understand how information on the raindrop size distribution can be obtained from the measurements, we write the drop diameter distribution, $N(D, s)\left[\mathrm{m}^{-3} \mathrm{~mm}^{-1}\right]$, at $\mathrm{r}=\mathrm{s}$, as

$$
N(D, s)=N_{t}(s) n(D, s)
$$

where $N_{t}$ is the number concentration $\left(\mathrm{m}^{-3}\right)$. For the lognormal distribution $n(D, s)$ can be expressed as [10]:

$$
n_{L N}(D ; \eta, \sigma)=(1 / \sqrt{2 \pi} \sigma D) \exp \left[(\ln D-\eta)^{2} / 2 \sigma^{2}\right]
$$

and for the Gamma distribution as [11]: 


$$
n_{G}(D ; \mu, \Lambda)=\left[\Lambda^{(\mu+1)} D^{\mu} / \Gamma(\mu+1)\right] \exp [-\Lambda D]
$$

where, in general, $\eta, \sigma$ for the log-normal distribution and $\Lambda, \mu$ for the Gamma distribution are functions of height. In the numerical results presented later, we use the median mass diameter, $D_{0}$, where $[10,11]$ :

$$
\Lambda D_{0}=3.67+\mu
$$

for the Gamma distribution and

$$
D_{0}=\exp \left[\eta+3 \sigma^{2}\right]
$$

for the log-normal distribution.

In the following equations we assume the Gamma parameterization; equations for the log-normal parameterization can be obtained by replacing $n_{G}(D ; \mu, \Lambda)$ with $n_{L N}(D ; \eta, \sigma)$. The specific attenuation from precipitation, $k_{p}$, can be expressed in terms of the drop diameter distribution $N(D, s)$ and the extinction cross section, $\sigma_{e}(f, D)$, by

$k_{p}(f, s)=c_{k} N_{t}(s) \int_{D} \sigma_{e}(f, D) n_{G}(D ; \mu, \Lambda, s) d D$

Noting that $k_{p}(f, s)$ is in $\mathrm{dB} \mathrm{km}^{-1}$ and taking $\sigma_{e}(f, D)$ to be in $\mathrm{mm}^{2}$ and $N_{t}$ in $\mathrm{m}^{-3}$ then $c_{k}=4.343 \times 10^{-3}$. Substituting (17) into (11) gives

$$
\delta A_{n}\left(f_{j}, f_{i}\right) \cong c_{k} \int_{0}^{r_{s}} N_{t}(s)\left\{\int_{D} n_{G}(D ; \mu, \Lambda, s) F_{n}\left(f_{j}, f_{i} ; D\right) d D\right\} d s
$$

where

$$
F_{n}\left(f_{j}, f_{i} ; D\right)=\alpha_{n}^{-1}\left(f_{j}, \bar{T}\right) \sigma_{e}\left(f_{j}, D\right)-\alpha_{n}^{-1}\left(f_{i}, \bar{T}\right) \sigma_{e}\left(f_{i} ; D\right)
$$

Although not written as such, $F_{n}\left(f_{j}, f_{i} ; D\right)$ varies with height because of the temperature dependence of $\sigma_{e}(f, D)$. Just as it is necessary to evaluate $\alpha_{n}$ at some mean cloud droplet temperature, a mean raindrop temperature must be used to evaluate $F_{n}\left(f_{j}, f_{i} ; D\right)$ in the estimates. Implicit in the equations below is the assumption that $F_{n}\left(f_{j}, f_{i} ; D\right)$ is independent of height and that, for the estimation procedure, the extinction cross sections must be computed at a fixed temperature. Errors that arise from this will be discussed in section 5 .

An interchange in the order of integration in (18) gives $\delta \mathrm{A}_{n}\left(f_{j}, f_{i}\right) \cong c_{k} \int_{D} F_{n}\left(f_{j}, f_{i} ; D\right)\left\{\int_{0}^{r_{s}} N_{t}(s) \dot{n}_{G}(D, \mu, \Lambda, s) d s\right\} d D$

Replacing the inner integral with the approximation:

$$
\bar{N}_{t} n_{G}(D ; \mu, \bar{\Lambda}) \cong r_{s}^{-1} \int_{0}^{r_{s}} N_{t}(s) n_{G}(D ; \mu, \Lambda, s) d s
$$

allows (18) to be written:

$$
\delta A_{n}\left(f_{j}, f_{i}\right) \cong c_{k} r_{s} \bar{N}_{t} \int n_{G}(D ; \mu, \bar{\Lambda}) F_{n}\left(f_{j}, f_{i} ; D\right) d D
$$

Although $\bar{N}_{t}$ and $\bar{\Lambda}$ yield the path-averaged number concentration and slope parameter only for range-independent drop size distributions, these will be taken to be the estimated values of these quantities.

If we have access to brightness temperatures measured at 3 frequencies, then, using the approximation (22), the ratio $\delta A_{n}\left(f_{3}, f_{1}\right) / \delta A_{n}\left(f_{2}, f_{1}\right)$ (or $\delta A_{n}\left(f_{3}, f_{1}\right) / \delta A_{n}\left(f_{3}, f_{2}\right)$ ) yields a quantity independent of the mean number concentration and approximately independent of cloud water:

$$
\frac{\delta A_{n}\left(f_{3}, f_{1}\right)}{\delta A_{n}\left(f_{2}, f_{1}\right)} \cong \frac{\int n_{G}(D ; \mu, \bar{\Lambda}) F_{n}\left(f_{3}, f_{1} ; D\right) d D}{\int n_{G}(D ; \mu, \bar{\Lambda}) F_{n}\left(f_{2}, f_{1} ; D\right) d D}
$$

If $\mu$ is a constant or a function of $\bar{\Lambda}$, then (23) can be solved numerically for $\bar{\Lambda}$ or $\bar{D}_{0}$. Once $\bar{\Lambda}$ has been found, $\bar{N}_{t}$ follows from (22). Next, $k_{p}$ can be estimated from $\bar{N}_{t}$ and $\bar{\Lambda}$ : this determines the first term of (8). Estimating $A(f)$ from $T_{B}(f)$ and using estimates of $\bar{N}_{t}$ and $\bar{\Lambda}$ from (22) and (23), respectively, provides the integrated cloud water content, $I_{M}$. Specifically, from (8), with $q_{n}$ in (7) taken to be unity, an estimator of $I_{M}$ is:

$$
\begin{aligned}
& I_{M}=\int_{0}^{r_{s}} M_{c}(s) d s=\alpha_{n}^{-1}(f, \bar{T})[\tilde{A}(f)- \\
& \left.c_{k} r_{s} \bar{N}_{t} \int_{D} n_{G}(D ; \mu, \bar{\Lambda}) \sigma_{e}(D, f) d D\right]
\end{aligned}
$$

where $f$ can be any of the frequencies at which the brightness temperature is measured. 
An estimate of the path-averaged rain rate $\left(\mathrm{mm} \mathrm{h}^{-1}\right)$ follows from the definition of this quantity along with $\bar{N}_{t}$ and $\bar{\Lambda}$ obtained from the previous equations:

$$
\bar{R}=c_{R} \bar{N}_{t} \int_{D} n_{G}(D ; \mu, \bar{\Lambda}) v(D) D^{3} d D
$$

where the velocity distribution of raindrops, $v(D)\left(\mathrm{m} \mathrm{s}^{-1}\right)$ is approximated by [12]:

$$
v(D)=9.25\left[1-\exp \left(-0.068 D^{2}-0.488 D\right)\right]
$$

where $\mathrm{D}$ is in $\mathrm{mm}$ in (25) and (26) so that

$$
c_{R}=0.6 \pi \times 10^{-3}
$$

As shown by the equations above, measurements of path attenuation are needed at a minimum of 3 frequencies to estimate the path-averaged rain rate and the parameters $\bar{N}_{t}$ and $\bar{\Lambda}$ of the drop size distribution. Although (23), which provides an estimate of $\bar{D}_{0}$, is independent of the effective range, $r_{s}$, through the precipitation, this is not the case for $\bar{N}_{t}$ and $\bar{R}$. In the case of stratiform rain, the effective range would include the melting layer and rain but not the dry snow above the melting layer, an estimate of which would require either the detection of the melting layer by radar or an estimate of the surface temperature and lapse rate.

\section{Conversion of Brightness Temperature to Path Attenuation}

To use the equations given in the previous section, the brightness temperatures must be related to the PIA or differential PIA. We begin by relating the brightness temperature, $T_{B}(f)$, to the PIA by $[13,14]$ :

$$
\tilde{A}(f)=c_{0}(f)+c_{1}(f) \ln \left[T_{0}-T_{B}(f)\right]
$$

where $T_{0}$ is a constant brightness temperature. Recalling that the differential normalized PIA, $\delta A_{n}\left(f_{j}, f_{i}\right)$, is defined by (9), and letting

$$
y=\delta A_{n}\left(f_{j}, f_{i}\right)
$$

then (28) implies that $y$ can be expressed in the following form

$$
y=\xi_{0}+\xi_{1} x_{1}+\xi_{2} x_{2}
$$

where $x_{1}=\ln \left[T_{0}-T_{B}\left(f_{j}\right)\right]$

$x_{2}=\ln \left[T_{0}-T_{B}\left(f_{i}\right)\right]$

Although the $\xi$ coefficients in (30) can be expressed in terms of $c_{0}$ and $c_{1}$ in (28), a more accurate procedure is to generate $A\left(f_{i}\right), T_{B}\left(f_{i}\right) ; i=1,2,3$ from model storms and determine the $\xi$ and c coefficients by linear regressions.

\section{Storm Model and Brightness Temperature Calculations}

We construct a simple model of stratiform rain by the following procedure [6]. From ground-based disdrometermeasured drop size distributions the number concentration and median mass drop diameter, $\mathrm{D}_{0}$, are calculated. Next, a bestfit $\mu$ is computed either for each DSD or for the ensemble. For the results here, we use $\mu=2$. As can be seen from (14) and (15), this information specifies a gamma distribution of drop diameters which is then used to characterize the distribution along an entire vertical column of precipitation. The procedure is repeated for a series of measured drop size distributions so that a set of such gamma distributions (and the corresponding vertical columns) are generated. The height of the rain layer is fixed at $4 \mathrm{~km}$; above this, a melting layer and snow layer are appended. In the melting region, the model of Yokoyama and Tanaka [15] is used along with the effective medium or Bruggeman approximation [16] for the effective dielectric constant of mixed-phase hydrometeors. Above the $0^{0}$ isotherm a $1 \mathrm{~km}$ layer of snow is added where the particle mass density is taken to be either $0.05 \mathrm{~g} \mathrm{~cm}^{-3}$ or $0.2 \mathrm{~g} \mathrm{~cm}^{-3}$. Throughout the vertical column the mass flux, or equivalent rain rate, is taken to be constant. Added to the precipitationsized particles is cloud liquid water which is specified by the liquid water content $\left(\mathrm{g} \mathrm{m}^{-3}\right)$ as a function of height. For the results presented here, a uniform distribution of cloud water of $1 \mathrm{~km}$ depth is located above the $0^{0}$ isotherm. $M_{c}$ is allowed to take on values from 0 to $2 \mathrm{~g} \mathrm{~m}^{-3}$ so that the integrated cloud water can range from 0 to $2 \mathrm{~kg} \mathrm{~m}^{-2}$. The relative humidity is allowed to take on values between $70 \%$ and $100 \%$. In the radiative transfer model of Kummerow [17], the surface emissivity is computed as a function of wind speed and frequency using the models of Hollinger et al. [18] and Stogyrn [19]. In the baseline model, we take the wind speed to be $7 \mathrm{~m} \mathrm{~s}^{-1}$; the effects of changes from this value are discussed later.

From the storm and surface scattering models, brightness temperatures and total path attenuations at various frequencies are calculated over each vertical column. The brightness temperatures are calculated using the Eddington approximation for the radiative transfer equation [17]. Although arbitrary incidence angles can be used, we restrict the calculations to nadir incidence. 


\section{Results}

Using the storm model just described, we calculate the apparent radar reflectivity factor profiles, $d B Z_{m}=10 \log _{10} Z_{m}$, at $9.5 \mathrm{GHz}$ for nadir incidence above the storm where the apparent, $Z_{m}$, and actual radar reflectivity factor, $Z$, are related at radar range $\mathrm{r}$ by

$$
Z_{m}(r)=Z(r) \exp \left[-0.2 \ln 10 \int_{0}^{r} k(s) d s\right] \text {. }
$$

A sequence of 379 profiles is shown in the top panel of Fig. 1. In this case, the integrated cloud water is taken to be $0.5 \mathrm{~kg} \mathrm{~m}^{-2}$ with snow density of $0.2 \mathrm{~g} \mathrm{~cm}^{-3}$ and $80 \%$ relative humidity. The radar bright-band, corresponding to mixed-phase hydrometeors in the melting layer, is evident in the region just below $4 \mathrm{~km}$. In the second panel from the top, the reflectivity factor difference,

$$
\delta Z_{m}(12,9.5 G H z)=d B Z_{m}(12 G H z)-d B Z_{m}(9.5 G H z)
$$

is shown. The characteristics of the reflectivity and differential reflectivity profiles are discussed in [6]. The corresponding brightness temperature results are shown in the lower two panels: $T_{B}(f=9.5 \mathrm{GHz})$ and $\delta T_{B}(12 G H z, 9.5 G H z)=T_{B}(12 G H z)-T_{B}(9.5 G H z)$.

Notice that $\delta T_{B}$ is bounded below at approximately $6 \mathrm{~K}$. The bulk of this difference is caused by the differential path attenuation from cloud liquid water, water vapor and molecular oxygen. If the integrated cloud water is set to zero the minimum brightness temperature difference is about $4 \mathrm{~K}$; if it is set to $1 \mathrm{~kg} \mathrm{~m}^{-2}$, the minimum $\delta T_{B}$ is about $8 \mathrm{~K}$. In other words, at light rain rates, an increase in the integrated cloud water of $1 \mathrm{~kg} \mathrm{~m}^{-2}$ results in an increase in the differential brightness temperature between 9.5 and $12 \mathrm{GHz}$ of about $4 \mathrm{~K}$.

To estimate $N_{t}, \bar{D}_{0}$ and $\bar{R}$ from (22), (23), and (25) it is first necessary to convert the $T_{B}$ to $A$ and $\delta A$ using the functional forms given by (28) and (30) for the storm model data shown in Fig. 1. For all fits, we assume that $T_{0}=280 \mathrm{~K}$. Tables 1 and 2 provide the coefficients $c_{0}, c_{1}$ and $\xi_{0}, \xi_{1}$, and $\xi_{2}$, respectively for several frequencies and frequency pairs. Plots of path attenuation versus brightness temperature are shown in Fig. 2 for the frequencies $9.5,10$ and $12 \mathrm{GHz}$ along with the fits (solid lines) using the coefficients from Table 1. It should be pointed out that the solution set of (30) defines a plane in y$\mathrm{x}_{1}-\mathrm{x}_{2}$ space: the solid curve shown in Fig. 3 is obtained by first expressing $T_{B}(f=12 \mathrm{GHz})$ as a linear function of $T_{B}(f=9.5 \mathrm{GHz})$ and then plotting $\mathrm{y}$ in (30) as a function of $T_{B}(f=9.5 \mathrm{GHz})$. It should also be noted that in Fig. 3 and for the coefficients in Table 2 we have chosen the first normalization factor so that $\delta A_{n}=\delta A_{1}$. This is also the normalization used for the $\bar{D}_{0}$ estimates.
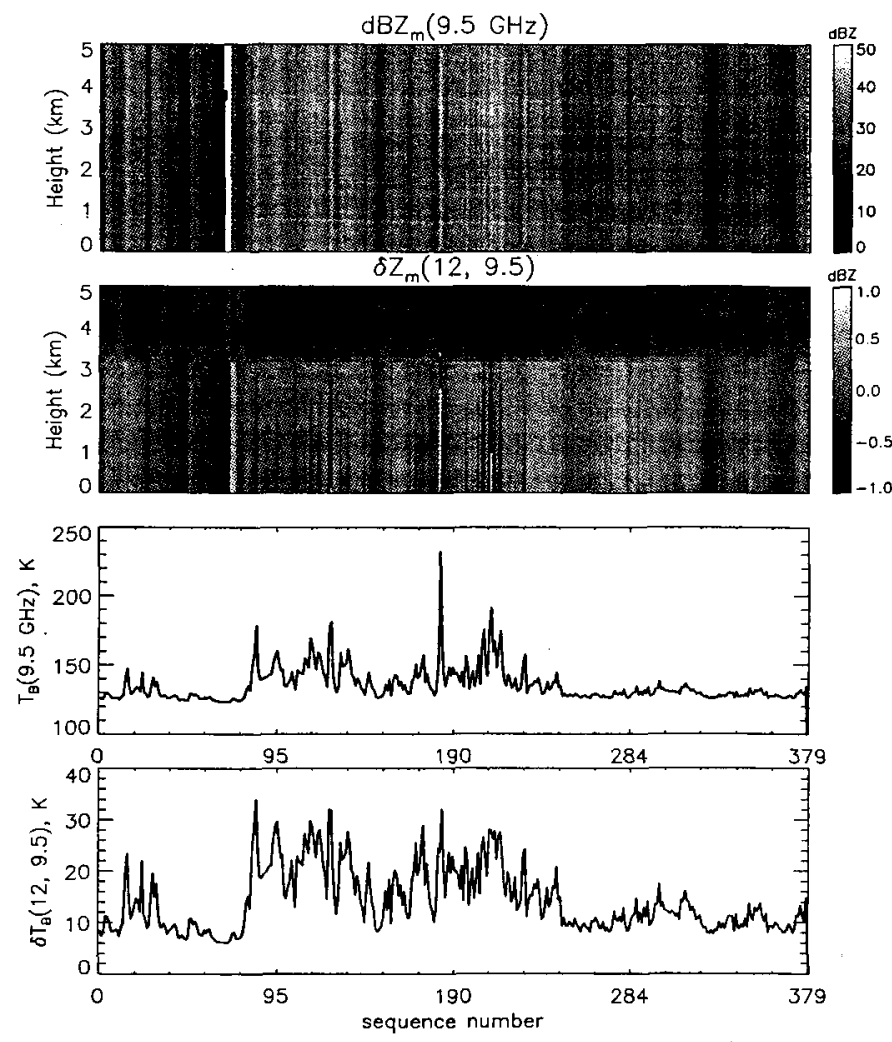

Fig. 1: Vertical profiles of measured radar reflectivity factor, $Z_{m}(9.5 \mathrm{GHz})$ (top); profiles of differential radar reflectivity factor, $Z_{m}(12 \mathrm{GHz}, 9.5 \mathrm{GHz}$ ), (second from top); brightness temperature, $T_{B}(9.5 \mathrm{GHz})$ (third from top), and differential brightness temperature, $T_{B}(12 \mathrm{GHz})-T_{B}(9.5 \mathrm{GHz})$ (bottom) for the baseline storm and surface model.
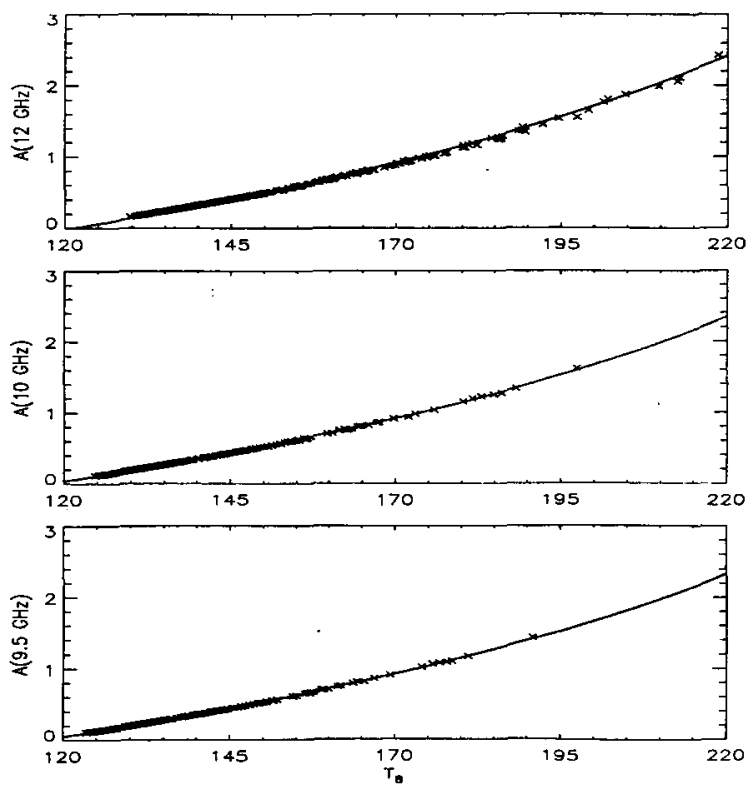

Fig. 2: Path-integrated attenuation (unnormalized) (dB) at 3 frequencies versus corresponding brightness temperature, $T_{B}$, using the baseline stratiform storm model. 


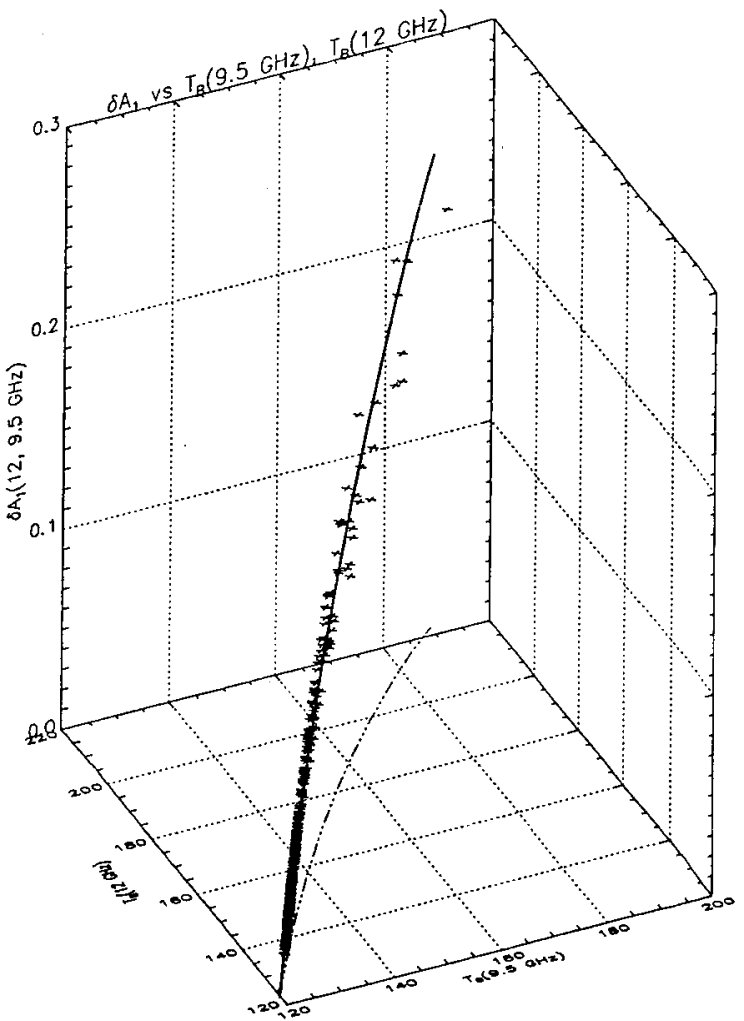

Fig. 3: $\delta A_{1}(12,9.5 \mathrm{GHz})$ versus $T_{B}(12 \mathrm{GHz})$ and $T_{B}(9.5$ $\mathrm{GHz}$ ) where values are derived from model data shown in Fig. 1 ; solid line represents a fitted curve through the data.

Table 1: Coefficients $c_{0}(f), c_{1}(f)$ in

$$
\tilde{A}(f)=c_{0}(f)+c_{1}(f) \ln \left[T_{0}-T_{B}(f)\right] ; T_{0}=280 \mathrm{~K}
$$

\begin{tabular}{lll}
\hline Frequency $(\mathrm{GHz})$ & $\mathrm{c}_{0}$ & $\mathrm{c}_{1}$ \\
\hline 9.5 & 11.89 & -2.33 \\
\hline 10 & 11.99 & -2.35 \\
\hline 11 & 12.25 & -2.40 \\
\hline 12 & 12.63 & -2.48 \\
\hline \hline
\end{tabular}

Table 2: $\xi$ coefficients, with $T_{0}=280 \mathrm{~K}$

$$
\delta A_{1}\left(f_{j}, f_{i}\right)=\xi_{0}+\xi_{1} \ln \left(T_{0}-T_{B}\left(f_{j}\right)+\xi_{2} \ln \left(T_{0}-T_{B}\left(f_{i}\right)\right)\right.
$$

\begin{tabular}{llll}
\hline$\left(f_{j}, f_{i}\right) \mathrm{GHz}$ & $\xi_{0}$ & $\xi_{1}$ & $\xi_{2}$ \\
\hline$(10,9.5)$ & 0.019 & -0.137 & 0.133 \\
\hline$(11,9.5)$ & 0.093 & -0.115 & 0.096 \\
\hline$(12,9.5)$ & 0.216 & -0.094 & 0.051 \\
\hline$(11,10)$ & 0.065 & -0.104 & 0.091 \\
\hline$(12,10)$ & 0.168 & -0.085 & 0.052 \\
\hline$(12,11)$ & 0.085 & -0.063 & 0.046 \\
\hline \hline
\end{tabular}
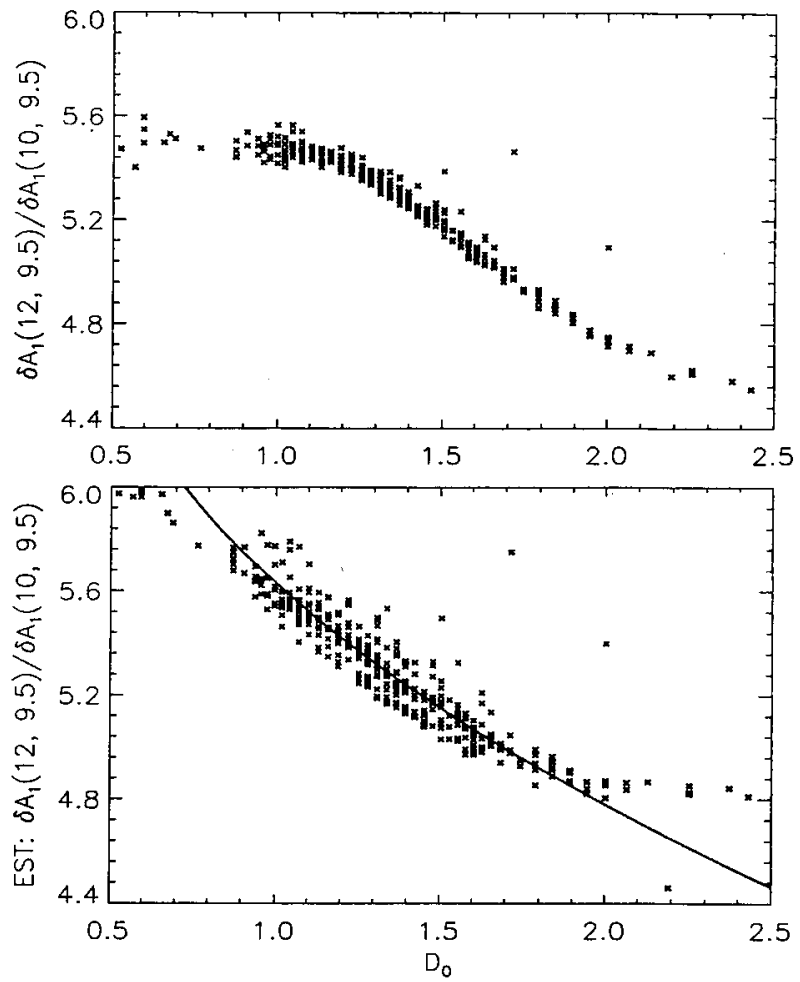

Fig. 4: Ratio of frequency-normalized differential pathattenuations (top) and corresponding estimate of this quantity derived from brightness temperatures (bottom). Results are plotted as a function of the median mass diameter, $D_{0}$. The solid line represents the best fit curve of $D_{0}$ as a function of the ratio plotted along the ordinate.

The coefficients given in Tables 1 and 2 are fixed for all calculations. Although these are the best-fit coefficients for the baseline model results, they are not the best-fit coefficients for models with changes in cloud water, water vapor, snow density or surface wind speed. Clearly, the feasibility of the method depends not only on the quality of estimates using data from a particular model but the accuracy of estimates from storm models that differ from the baseline.

Using (23), we compute and plot in Fig. 4 the ratio $p=\delta A_{1}\left(f_{3}, f_{1}\right) / \delta A_{1}\left(f_{2}, f_{1}\right) \quad$ versus $\quad \bar{D}_{0} \quad$ where $\left(f_{1}, f_{2}, f_{3}\right)=(9.5,10,12) G H z$. For the scatter plot in the top panel, the true differential normalized path integrated attenuation is used to compute the ratio while for the bottom panel $\delta A_{1}\left(f_{3}, f_{1}\right)$ and $\delta A_{1}\left(f_{3}, f_{2}\right)$ are estimated from the brightness temperatures using (30) with the coefficients listed in Table 2. Differences between the upper and lower scatter plots are largely due to errors incurred in converting brightness temperatures to $\delta A_{1}$. Notice also that at small $\bar{D}_{0}$, (typically light rain rates), the true value of $p$ is determined primarily by the differential attenuations of the cloud and atmospheric 
gases and is nearly independent of the characteristics of the precipitation. Taking the data in the bottom panel of Fig. 4 as the basis of estimating $\bar{D}_{0}$ from $p$, then on using a quadratic fit to the data, we obtain:

$$
\bar{D}_{0}=17.02-4.81 p+0.350 p^{2}
$$

where (35) is plotted as a solid line in the lower panel of Fig. 4.

The results shown in Figs. 1-4 address the forward problem where the brightness temperature and path-integrated attenuation is computed for a given frequency and storm model. The remainder of the paper is focused on the inverse problem where estimates of rain rate and parameters of the drop size distribution are obtained from brightness temperatures at several frequencies.

To obtain an estimate of $\bar{D}_{0}$, we first convert the $T_{B}$ to $\delta A_{1}\left(f_{3}, f_{1}\right)$ and $\delta A_{n}\left(f_{3}, f_{2}\right)$ using (30) and the coefficients in Table 2. From these quantities $p$ is computed. $\bar{D}_{0}$ then follows from (35). The results of the procedure are shown in Fig. 5 (top). Once $\bar{D}_{0}$ is obtained, $\bar{N}_{t}$ is determined from (22). A scatter plot of the logarithm (base 10) of the estimated $\bar{N}_{t}$ values versus the 'true' values (i.e., those values used in the forward calculations of $T_{B}$ and $A$ ) is shown in the bottom panel of Fig. 5 . Less scatter in the $\bar{N}_{t}$ estimates is achieved by using $\delta A_{2}$ rather than $\delta A_{1}$ so that the $n=2$ normalization in (22) has been used. It worth emphasizing that although (35) is the best-fit curve of $\bar{D}_{0}$ versus $p$ for the data generated from the baseline model only, the coefficients of the fit are used for all subsequent estimates despite changes in the storm model parameters and wind speed used in the calculations of $T_{B}$.

From the estimated values of $\bar{D}_{0}$ and $\bar{N}_{t}$, shown in Fig. 5 , the rain rate estimates are calculated from (25). Plots of the results versus sequence number are shown in the top panel of Fig.6; below this are plotted the true rain rates. A scatter plot of the estimated versus true rain rates is given in the bottom panel of Fig. 6.

As mentioned in section 3, evaluation of the integrals in (22) (25) requires computations of $\sigma_{e}(f, D)$ and $F_{n}\left(f_{j}, f_{i} ; D\right)$ at a fixed temperature. For the results in Figs. 4 and 5, the near-surface temperature of $24 \mathrm{C}$ was used. If we change this to the temperature of the midpoint of the rain layer $(12 \mathrm{C})$, we obtain almost identical results for $\bar{D}_{0}$ but significantly different values for $\bar{N}_{t}$ and $\bar{R}$. Plots of the estimated versus true values of these latter two variables are shown in Fig. 7; it
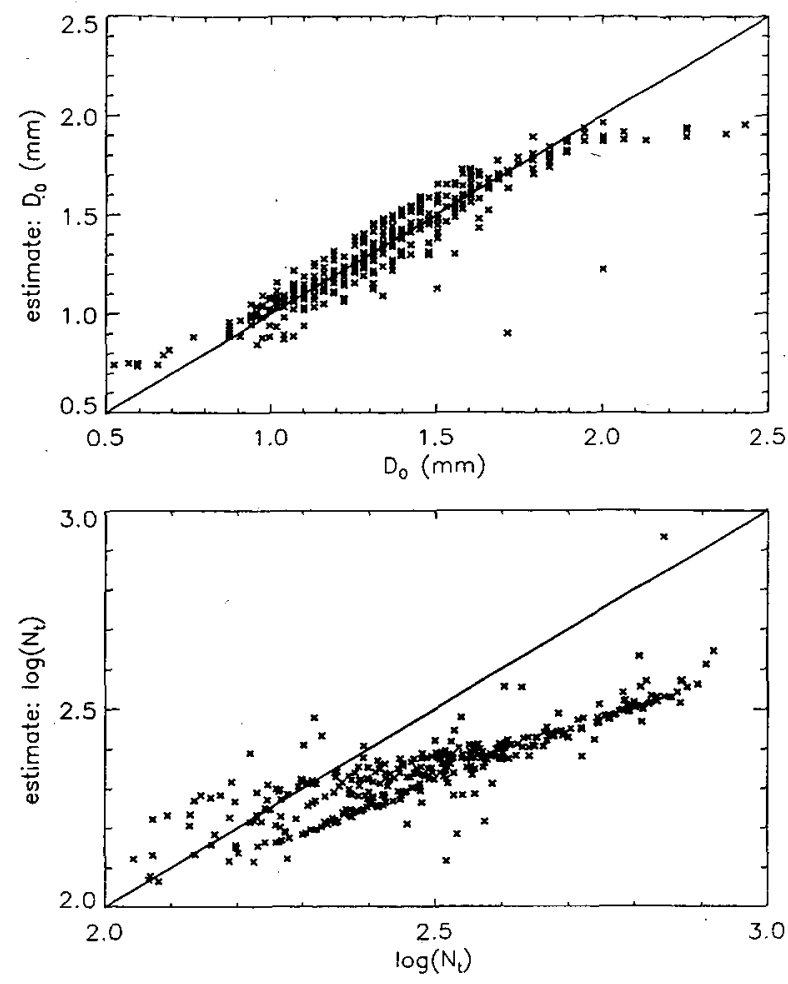

Fig. 5: Top: Estimated median mass diameter $\left(D_{0}\right)$ versus the true value using the fitting curve shown in Fig. 4. Bottom: logarithm of estimated number concentration, $N_{t}$.
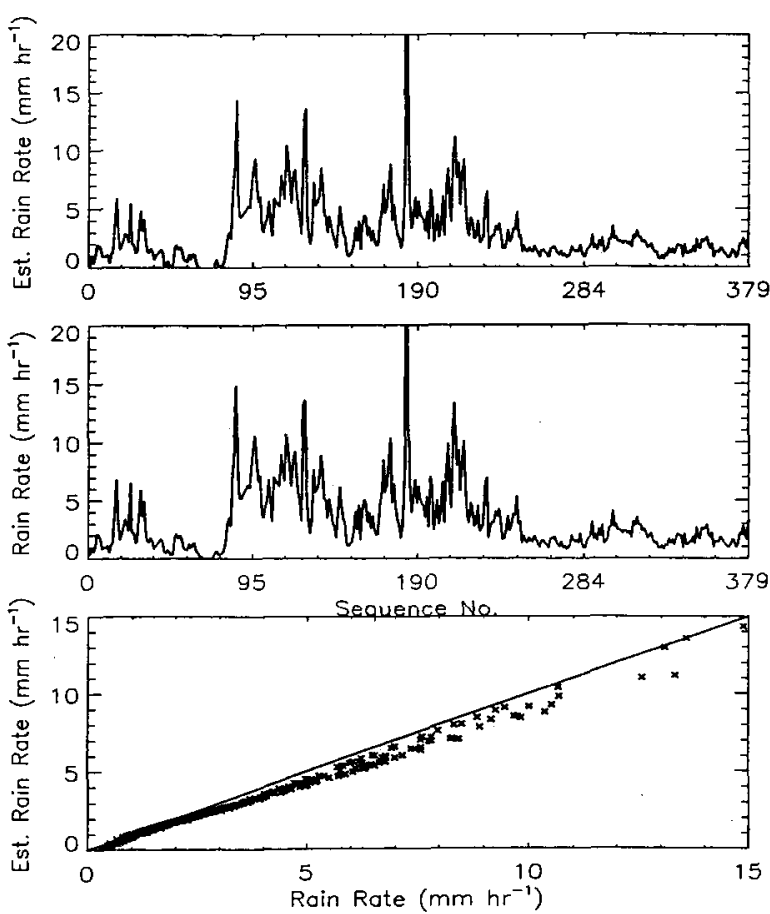

Fig. 6: Top: Estimated and true rain rates versus sequence number. Bottom: Scatter plot of estimated and true rain rates from data in the top panels. 
can be seen that a change in the assumed temperature of raindrops from 24 to $12 \mathrm{C}$ increases the estimates of number concentration and produces positively biased rain rates. For subsequent plots and computations, $\sigma_{e}(f, D)$ and $F_{n}\left(f_{j}, f_{i} ; D\right)$ are evaluated at $24 \mathrm{C}$.
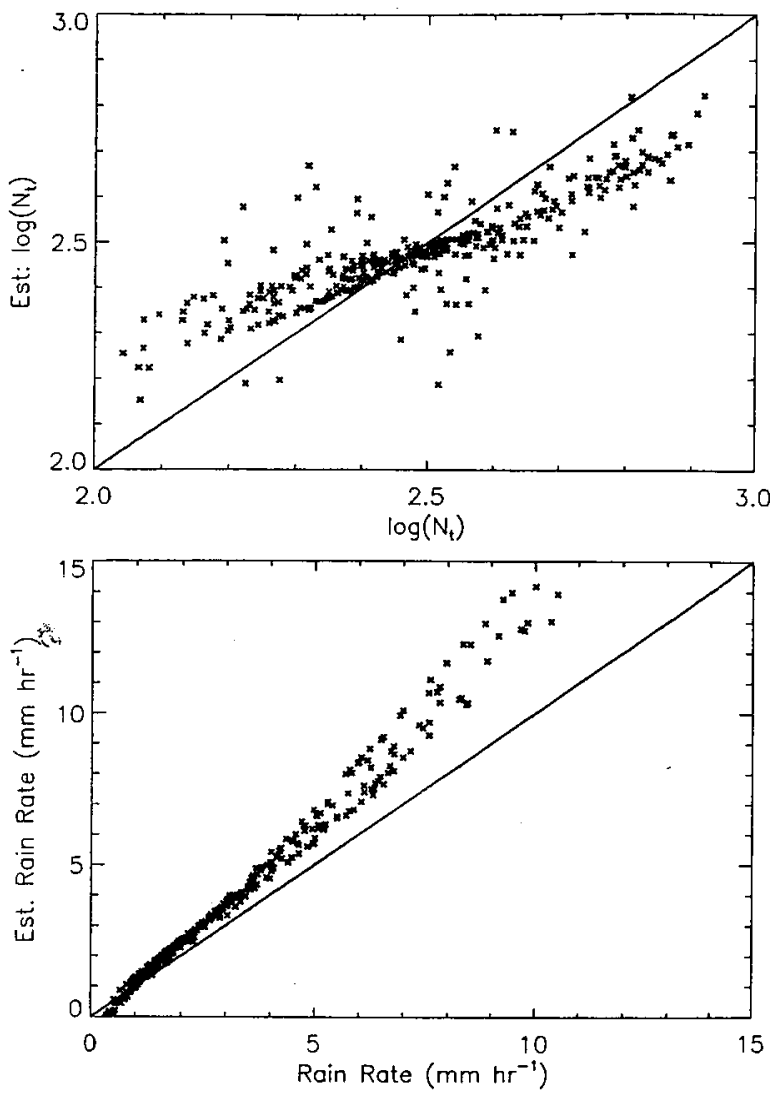

Fig. 7: Effects of a change in temperature from $24 \mathrm{C}$ to $12 \mathrm{C}$ at which estimates are evaluated. Top: Estimated versus true values of $\log \left(\mathrm{N}_{\mathrm{v}}\right)$; Bottom: estimated versus true values of rain rate.

Before discussing changes to the baseline model, we note that estimates of integrated cloud water using (24) are generally unreliable. The reason for this can be understood by recognizing that (24) expresses the cloud water attenuation, $\alpha_{c}\left(f, \overline{T_{c}}\right) I_{M}$, as a difference between the total attenuation and the attenuation from precipitation alone. The estimate is positively biased because attenuation contributions from water vapor and $\mathrm{O}_{2}$ have not been subtracted from the total. This problem can be partly circumvented by modifying the estimate to include this subtraction using nominal values for these contributions. However, a more serious problem with (24) is that for moderate and heavy rain rates, the cloud water attenuation, for the models considered here, is a small fraction of the total. As a consequence, variations in the estimate of the second term on the right-hand side of (24) introduce large errors in $I_{M}$ because of the subtraction of quantities of roughly equal magnitude.
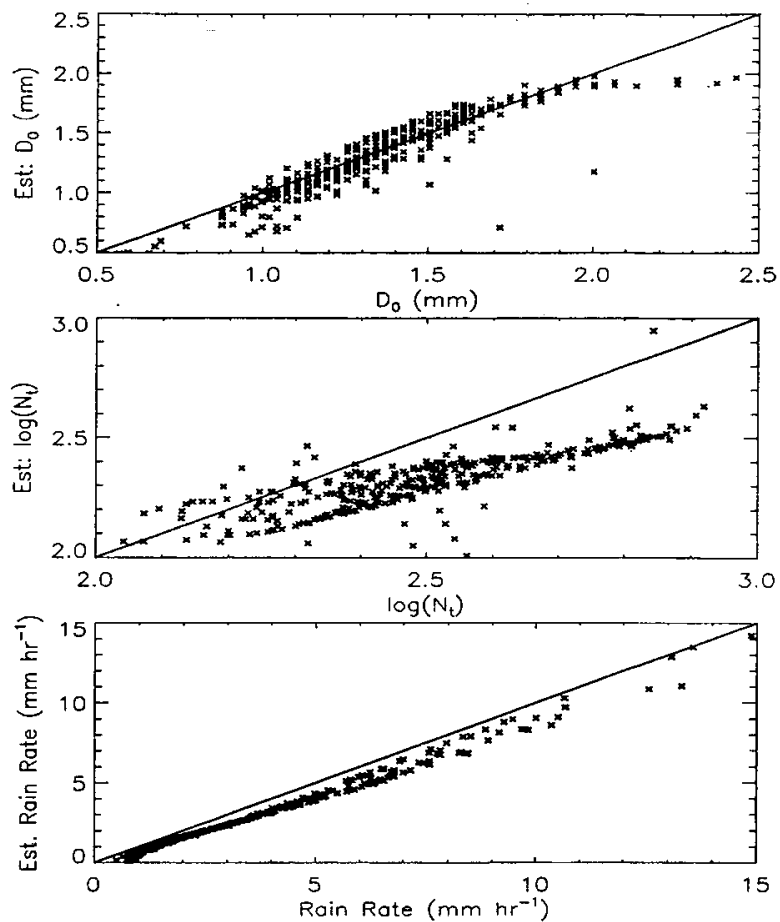

Fig. 8: Estimated versus true values of $D_{0}, \log \left(N_{t}\right)$ and $R$ for a modified stratiform storm model: snow density $=0.2 \mathrm{~g} \mathrm{~cm}^{-3}$, integrated cloud water $=0.25 \mathrm{~kg} \mathrm{~m}^{-2}, \mathrm{RH}=90 \%$.
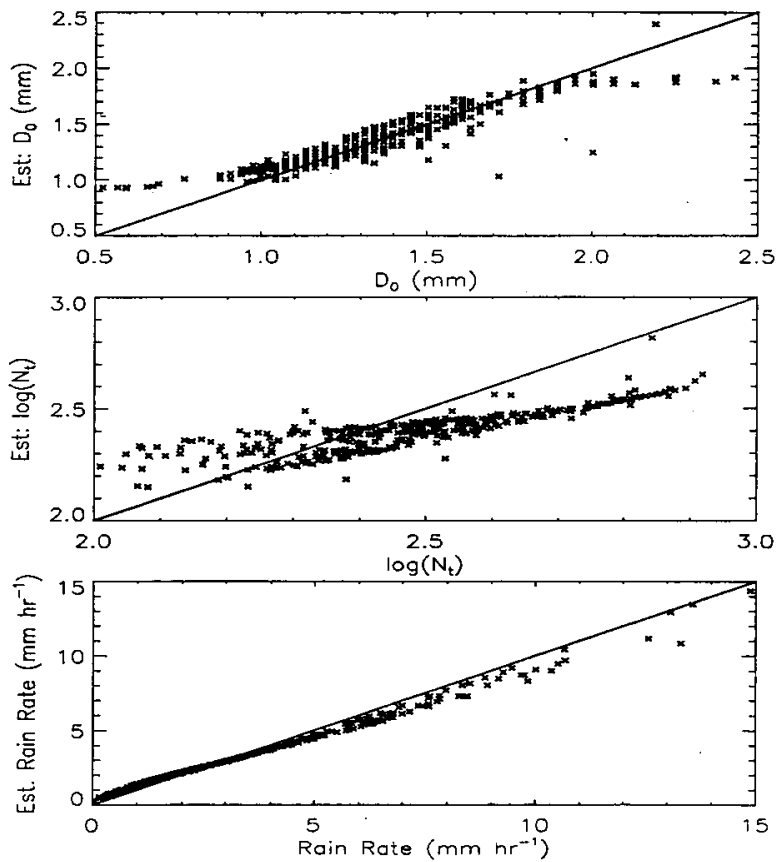

Fig. 9: Estimated versus true values of $D_{0}, \log \left(N_{t}\right)$, and $R$ for a modified stratiform storm model: snow density $=0.05 \mathrm{~g} \mathrm{~cm}^{-3}$, integrated cloud water $=0.75 \mathrm{~kg} \mathrm{~m}^{-2}, \mathrm{RH}=90 \%$. 
The remaining examples focus on results from modifications of the baseline stratiform storm model. Shown in Figs. 8-10 are scatter plots of the estimated versus true values of $\bar{D}_{0}$ (top), $\log \left(\bar{N}_{t}\right)$ (center) and $\mathrm{R}$ (bottom) for the sets of model parameters in Table 3. (It is worth noting that a replacement of the effective medium with the Maxwell Garnet formulation, water matrix with ice inclusions, for melting snow particles produces a significant increase in brightness temperatures which leads to increases in $N_{t}$ and positive biases in the rain rate. The results are somewhat similar to those shown in Fig. 7. A detailed study of the effects of the mixed-phase region is beyond the scope of the paper, however.)

Table 3: Parameters used in the calculation of brightness temperature

\begin{tabular}{llllll}
\hline Model & $\begin{array}{l}\text { Snow } \\
\text { density } \\
\left(\mathrm{g} \mathrm{cm}^{-3}\right)\end{array}$ & $\begin{array}{l}\text { Integrated } \\
\text { cloud } \\
\text { water } \\
\left(\mathrm{kg} \mathrm{m}^{-2}\right)\end{array}$ & $\begin{array}{l}\text { Relative } \\
\text { humidity } \\
(\%)\end{array}$ & $\begin{array}{l}\text { Ocean } \\
\text { wind } \\
\text { speed } \\
\left(\mathrm{m} \mathrm{s}^{-1}\right)\end{array}$ & $\begin{array}{l}\text { Relevant } \\
\text { figures }\end{array}$ \\
\hline baseline & 0.2 & 0.5 & 80 & 7 & $1-7$ \\
\hline Mod 1 & 0.2 & 0.25 & 90 & 7 & 8 \\
\hline Mod 2 & 0.05 & 0.75 & 90 & 7 & 9 \\
\hline Mod 3 & 0.2 & 2 & 80 & 7 & 10 \\
\hline Mod 4 & 0.2 & 0.5 & 80 & 15 & 11 \\
\hline
\end{tabular}
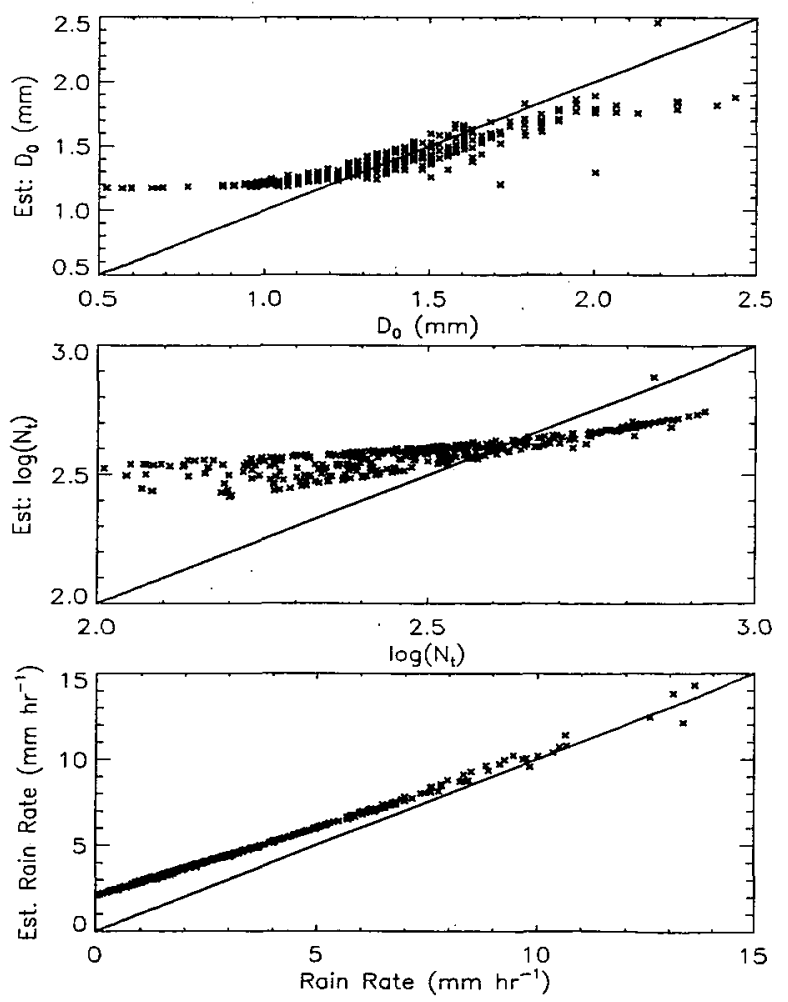

Fig. 10: Estimated versus true values of $D_{0}, \log \left(N_{t}\right)$, and $R$ for a modified stratiform storm model: snow density $=0.2 \mathrm{~g} \mathrm{~cm}^{-3}$, integrated cloud water $=2 \mathrm{~kg} \mathrm{~m}^{-2}, \mathrm{RH}=80 \%$.
Despite the fairly large variability in $\bar{D}_{0}$ and $\bar{N}_{t}$ in these examples, the rain rates tend to have a small amount of scatter relative to the true values with generally negative biases for cases shown in Figs. 8 and 9 and a positive bias for the case in Fig. 10. As noted earlier, the bias is also affected by the assumed temperature at which $\sigma_{e}(f, D)$ and $F_{n}\left(f_{j}, f_{i} ; \dot{D}\right)$ are evaluated and the type of normalization used to form the estimates. The reason for the relatively accurate rain rate estimates appears to arise from the fact that $\bar{D}_{0}$ and $\bar{N}_{t}$ are inversely correlated so that an overestimate in one is compensated by an underestimate in the other. This compensation works well for the rainfall estimate because of the underlying path attenuation constraint; that is, the brightness temperatures at the various frequencies are highly correlated with attenuation. Moreover, because rain rate and attenuation are approximately equal to the same moment of the drop size distribution [20], this constraint extends, in an
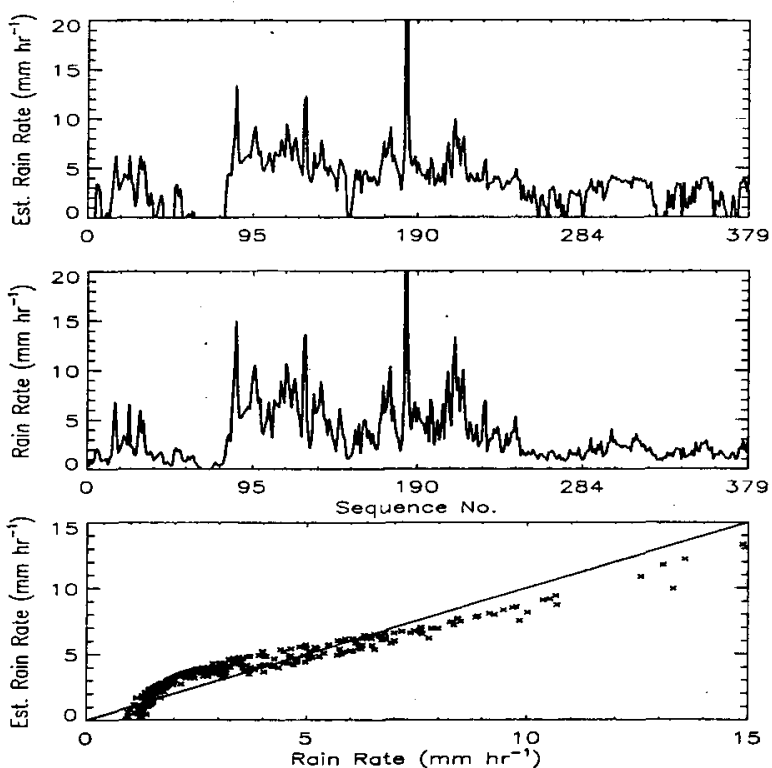

Fig 11: Estimated versus true rain rates for an ocean wind speed of $15 \mathrm{~m} \mathrm{~s}^{-1}$.

approximate sense, to the rain rate estimates. For similar reasons, a direct conversion of a brightness temperature measurement at X-band to path-averaged rainfall rate is fairly accurate. The primary advantage of the use of multiple frequencies is the added information provided on pathaveraged number concentration and median mass diameter. Although the rainfall estimates appear to be reasonably accurate, the improvement over a straightforward rain rate estimator using a single brightness temperature needs to be explored.

In previous examples, the $T_{B}$ were calculated under the assumption of an ocean wind speed of $7 \mathrm{~m} \mathrm{~s}^{-1}$. In the baseline model, the assumed wind speed was also taken to be $7 \mathrm{~m} \mathrm{~s}^{-1}$. If 
the $T_{B}$ are calculated at a higher wind speed than the assumed value of $7 \mathrm{~m} \mathrm{~s}^{-1}$, we find that $\bar{D}_{0}$ is positively biased and $\bar{N}_{t}$ is negatively biased. Fig. 11 shows the effect on the rain rate estimates for a wind speed of $15 \mathrm{~m} \mathrm{~s}^{-1}$, where, as always, a $7 \mathrm{~m}$ $\mathrm{s}^{-1}$ speed is assumed in the retrieval. Note that the relative error is particularly large at light rain rates. For low winds, the errors in the estimated quantities are much smaller. In general, the results suggest that errors are acceptable up to wind speeds of about $10 \mathrm{~m} \mathrm{~s}^{-1}$ to $15 \mathrm{~m} \mathrm{~s}^{-1}$ although accuracy depends on the magnitude of the rain rate and cloud liquid water as well as other error sources. Some improvement in the retrieval should be possible by estimating wind speed in nearby rain-free areas. However, in high-wind environments such as hurricanes, the algorithms are unstable and the technique devised for the SFMR becomes applicable [1,2].

\section{Summary and Conclusions}

Brightness temperature measurements at multiple frequencies within X-band, over a span of $2-3 \mathrm{GHz}$, may provide information on the path-averaged rainfall rate and parameters of the raindrop size distribution. The potential for this type of estimation arises from the fact that at X-band, brightness temperature is well correlated with path-integrated attenuation and that a difference of path attenuations normalized by frequency is nearly independent of cloud liquid water attenuation. Moreover, a ratio of such differences, derived from brightness temperatures at three X-band frequencies, provides an estimate of the median mass diameter, $D_{0}$, of the rain. The equations also lead to estimates of the mean number concentration, $\mathbf{N}_{t}$, and rainfall rate. A simple simulation using a stratiform storm model suggests that reasonably accurate rain rates are possible even though the variability in $D_{0}$ and $N_{t}$ can be high. On the other hand, estimates of integrated cloud water content appear to be unreliable. Although the approach appears to be feasible for low to moderate wind speeds over ocean, more detailed studies are needed on variations in the shape parameter, $\mu$, changes in the drop size distribution with height and the effects of the melting layer. The error budget must also include the measurement error of the brightness temperatures in the context of the system design, measurement requirements and scanning strategy.

\section{ACKNOWLEDGMENT}

I wish to thank Chris Kummerow for the use of his radiative transfer code and Jim Weinman for helpful discussions and suggestions.

\section{REFERENCES}

[1] P.G. Black and C.L. Swift, "Airborne stepped frequency microwave radiometer measurements of rainfall rate and surface wind speed in hurricanes," $22^{\text {nd }}$ Conf. Radar Meteorology, pp. 433-438, Sept., 1984, Amer. Meteor. Soc., Boston, MA.

[2] E.W. Uhlhorn and P.G. Black, "Verification of remotely sensed sea surface winds in hurricanes," J. Atmos. Oceanic Technol., vol. 20, pp. 99116, Jan. 2003.
[3] Y.T. Furuhama and T. Thara, "Remote sensing of path-average raindrop size distributions from microwave scattering measurements," IEEE Trans. Antennas Propagat., vol. AP-29, pp. 275-281, Mar. 1981.

[4] T. Thara, Y. Furuhama, and T. Manabe, "Inference of raindrop size distribution from rain attenuation statistics at 12,35 , and $82 \mathrm{GHz}$," Trans. IECE Japan, vol. E67, pp. 211-217, April 1984.

[5] R.F. Rincon and R.H. Lang, "Microwave link dual-wavelength measurements of path-average attenuation for the estimation of drop size distribution and rainfall," IEEE Trans. Geosci. Remote Sens., vol. GE-40, pp. 760-770, April 2002.

[6] G. Zhang, J. Vivekanandan, and E. Brandes, "A method for estimating rain rate and drop size distribution from polarimetric radar measurements," IEEE Trans. Geosci. Remote Sens., vol. 39, pp. 830-841.

[7] R. Meneghini, L. Liao, S. W. Bidwell, and G. M. Heymsfield, "On the feasibility of a Doppler weather radar for estimates of drop size distribution using two closely-spaced frequencies," IEEE Trans. Geosci. Remote Sens., vol. GE-39, Oct. 2001.

[8] R. Meneghini, S.W. Bidwell, L. Liao, R. Rincon, and G.M. Heymsfield, "Differential-frequency Doppler weather radar: theory and experiment," Radio Science, vol. 38, pp. 5.1-5.10, Mar. 2003.

[9] L.J. Battan, Radar Observations of the Atmosphere. The Univ. of Chicago Press, 1973, 324 pp.

[10] G. Feingold and Z. Levin, "The log-normal fit to raindrop spectra from frontal convective clouds in Israel," J. Climate Appl. Meteor., vol. 25, pp. 1346-1363, 1986.

[11] C.W. Ulbrich, "Natural variations in the analytical form of the raindrop size distribution," J. Climate Appl. Meteor., vol. 22, pp. 1764-1775, 1983.

[12] R. Lhermitte, "Cloud and precipitation sensing at $94 \mathrm{GHz}$," IEEE Geosci. Remote Sens., vol. GE-26, pp. 207-216, Jan. 1988.

[13] J.A. Weinman, R. Meneghini, and K. Nakamura, "Retrieval of precipitation profiles from airborne radar and passive radiometer measurements: comparison with dual-frequency radar measurements," $J$. Appl. Meteor., vol. 29, pp. 981-993, Oct. 1990.

[14] Durden et al., "Measurement of rainfall path attenuation near nadir: a comparison of radar and radiometer methods at $13.8 \mathrm{GHz}$," Radio Science, vol. 30, pp. 943-947, July-Aug. 1994.

[15] T. Yokoyama and H. Tanaka, "Microphysical processes of melting snowflakes detected by two-wavelength radar," J. Meteor. Soc. Japan, vol. 62, pp. 650-666, 1984.

[16] D.A.G. Bruggeman, "Berechnung vershiedener physikalischer Konstanten von heteorogenen Substanzen. I. Dielectrizitätskonstanten und Leitfähigkeiten der Mischkörper aus isotropen Substanzen," Ann. Phys., vol. 24, pp. 636-679, 1935.

[17] C. Kummerow, "On the accuracy of the Eddington Approximation for radiative transfer in the microwave frequencies," $J$. Geophys. Res., vol. 98, pp. 2757-2765, Feb. 1993.

[18] J. Hollinger, R. Lo, G. Poe, R. Savage, and J. Pierce, Special Sensor Microwave Imager User's Guide, 120pp., Naval Research Laboratory, Washington, D.C., 1987.

[19] A. Stogym, "The emissivity of sea foam at microwave frequencies," $J$. Geophys. Res., vol. 77, pp. 1658-1666, 1972.

[20] D. Atlas and C.W. Ulbrich, "Path- and area-integrated rainfall measurement by microwave attenuation in the $1-3 \mathrm{~cm}$ band," $J$. Appl. Meteor., vol. 16, 1322-1331, 1977. 